\title{
Summation and overexpectation with qualitatively different outcomes
}

\author{
ROBERT A. RESCORLA \\ University of Pennsylvania, Philadelphia, Pennsylvania
}

\begin{abstract}
In five experiments, a Pavlovian appetitive conditioning preparation was used with rats to explore the interaction of associations with different, but equivalently valued, outcomes. Experiment 1 demonstrated summation in magazine responding when two stimuli previously associated with different outcomes were combined. Experiments $2 \mathrm{~A}$ and $2 \mathrm{~B}$ found that pairing that stimulus compound with one of the outcomes led to a decrease in performance (overexpectation) for both of the stimulus elements. Experiments $3 \mathrm{~A}$ and $3 \mathrm{~B}$ confirmed that result but demonstrated the continued presence of the original stimulus-outcome associations. The results are consonant with a view in which replacing one outcome with another leads to an associative structure containing both stimulus-outcome associations and an outcome-independent depressive process.
\end{abstract}

There is now excellent evidence that once an association with an outcome has been learned, it is highly resistant to change. This appears to be true of both the stimulus-outcome ( $\mathrm{S}-\mathrm{O})$ associations learned in Pavlovian conditioning and the response-outcome $(\mathrm{R}-\mathrm{O})$ associations learned in instrumental training (see, e.g., Rescorla, 1998).

For instance, subjecting the association to extinction by the separate occurrence of either the $S$ or the $R$ produces behavioral decrement; but at the same time, it apparently leaves the associations with outcomes intact (see, e.g., Rescorla 1996a, 1996b). One can detect the continued presence of those original associations by using either a devaluation or a transfer procedure. In a devaluation procedure, reducing the value of an outcome selectively depresses both Rs and behavior to Ss previously associated with that outcome. This selective effect of devaluing an outcome occurs even after extinction, although in that case, of course, detection of the associations requires that the overt behavior first be artificially elevated by training with another outcome. In a transfer procedure, an $S$ will transfer control to a new $R$ to the degree that the $S$ and the $R$ were originally trained with a common outcome. This selective transfer persists even after either the $\mathrm{S}$ or the $\mathrm{R}$ has been subjected to extinction.

Similar results have been obtained when the identity of the outcome is changed during the course of conditioning. The replacement of one outcome by another, equivalently valued outcome that produces the same learned response leaves the association with the first outcome intact and adds an association with the second outcome. Again, devaluation and transfer procedures detect the pres-

This research was supported by National Science Foundation Grant IBN94-04676. Correspondence concerning this article should be addressed to R. A. Rescorla, Department of Psychology, University of Pennsylvania, 3815 Walnut Street, Philadelphia, PA 19104 (e-mail: rescorla@psych.upenn.edu). ence of these associations (see, e.g., Delamater, 1996; Rescorla, 1995, 1996a).

However, these experiments led to another observation that creates something of a puzzle. Those experiments found that the replacement of one outcome by another of equivalent value, capable of producing the same conditioned response, resulted in little change in overall performance. Yet, the devaluation and transfer procedures suggested that an association with the new outcome had been added without the removal of the association with the original outcome. Because the two outcomes are of similar value and control similar conditioned responses, one might have anticipated that this addition to the total associative strength would have produced a substantial increase in performance. But it did not. The present series of experiments explores a possible resolution to that puzzle.

The observation that removing the outcome during extinction eliminates the trained behavior, while leaving the underlying outcome associations largely unaffected, naturally raises the problem of accounting for the behavioral decrement. This has encouraged the supposition, originally suggested by Pavlov (1927), that extinction is accomplished not by the removal of the effects of training but rather by the superimposition of some new depressive process. The fact that this depressive process leaves information about the particular outcome intact suggests that it is relatively outcome independent.

Moreover, it seems possible that the same decremental process occurs when one outcome replaces another, if those outcomes are of similar value ands control the same response. This possibility is made more plausible by noting that shifting from $\mathrm{O}_{1}$ to $\mathrm{O}_{2}$ can be construed as conducting extinction on the $\mathrm{S}-\mathrm{O}_{1}$ or $\mathrm{R}-\mathrm{O}_{1}$ association while concurrently adding the $\mathrm{S}-\mathrm{O}_{2}$ or $\mathrm{R}-\mathrm{O}_{2}$ association. One could then characterize the associative changes resulting from a shift from one outcome to another as follows: When $\mathrm{S}-\mathrm{O}_{1}$ conditioning is followed by $\mathrm{S}-\mathrm{O}_{2}$ training, the or- 
ganism maintains a fully intact $\mathrm{S}-\mathrm{O}_{1}$ association and adds to it a full $\mathrm{S}-\mathrm{O}_{2}$ association. Ordinarily, those two associations would be expected to summate, leading to an increase in behavior. However, should that begin to happen, the total level of responding would be inappropriately large, since only one outcome actually occurs. The result is that there is an "overexpectation" that produces a behavioral decrement (see, e.g., Kamin \& Gaioni, 1974; Kremer, 1978; Rescorla, 1970; Wagner, 1971). This decrement is essentially similar to that observed in extinction. In the case of extinction, there is a discrepancy between the responding that prior reinforcement with $\mathrm{O}_{1}$ produces and that which nonreinforcement will support. In the case of a shift in outcome, there is a discrepancy between the responding that prior reinforcement with $\mathrm{O}_{1}$ and $\mathrm{O}_{2}$ produces and that which $\mathrm{O}_{2}$ alone will support. The occurrence of the decrement, which brings behavior back into line with the value of the outcome delivered, seems to be accomplished by a process that leaves both the $\mathrm{S}-\mathrm{O}_{1}$ and the $\mathrm{S}-\mathrm{O}_{2}$ intact but that separately depresses performance. The result is that, as behavior begins to increase with the addition of the $\mathrm{S}-\mathrm{O}_{2}$ association, a decremental process counteracts that increase. Consequently, the addition of the new $\mathrm{S}-\mathrm{O}_{2}$ association does not result in an overall increase in performance.

There is some indirect evidence to support the argument that a decremental process similar to that of extinction also occurs when $\mathrm{O}_{2}$ replaces $\mathrm{O}_{1}$. One well-documented property of the decremental process that produces extinction is spontaneous recovery, the partial restoration of performance with the passage of time (see, e.g., Mackintosh, 1974; Pavlov, 1927). If the same decremental process occurs when one outcome replaces another, we should expect to see a similar growth in responding with the passage of time after that manipulation. This increase in performance should occur despite the fact that no depression was produced by the replacement. A recent series of experiments has documented such an increase with time for the cases of instrumental and Pavlovian associations (Rescorla, 1996c, 1997a, 1997b).

The intention of the present experiments was to provide more direct evidence for each of the assumptions underlying this explanation of the behavior observed when one outcome is replaced by another. The experiments focused on the particular case of two outcomes that, although qualitatively distinct, had similar values and conditioned the same response. The present explanation depends on three separable claims: that summation should be observed when signals of different outcomes are presented in compound, that reinforcing such a compound with one of the outcomes should produce a decrement in performance, and that this decrement should be achieved independently of changes in the original S-O associations. In Experiment 1, the first claim, that one should anticipate the summation of associations with differing outcomes, was examined. In Experiments $2 \mathrm{~A}$ and $2 \mathrm{~B}$, the second claim, that this summation should result in overexpectation, which in turn produces behavioral decrement, was examined. In Exper- iments $3 \mathrm{~A}$ and $3 \mathrm{~B}$, the third claim, that this behavioral decrement should be outcome independent, leaving the original associations intact, was examined.

All of the experiments used a conventional Pavlovian conditioning procedure with rat subjects in which auditory and visual stimuli signaled the occurrence of positively valued outcomes. The associations are measured in terms of approach to the point of outcome delivery.

\section{EXPERIMENT 1}

The goal of this experiment was to assess the summation of associations based on the different outcomes used in previous experiments and to compare that with the summation of associations based on the same outcome. In order to ensure equivalent exposure to the different outcomes themselves, a within-subjects design was used, as is illustrated in Figure 1. In this design, each animal received Pavlovian conditioning with two auditory stimuli $\left(A_{1}\right.$ and $\left.A_{2}\right)$, a noise and a tone, and two visual stimuli $\left(V_{1}\right.$ and $\left.V_{2}\right)$, a steady diffuse houselight and a flashing localized light. One stimulus within each modality was paired with a solid pellet and the other with liquid sucrose (counterbalanced as $\mathrm{O}_{1}$ and $\mathrm{O}_{2}$ ). Then the animals were tested with each elemental stimulus and with all of the cross-modality compounds. Two of those compounds $\left(V_{1} A_{1}\right.$ and $\left.V_{2} A_{2}\right)$ provide the opportunity to observe summation of associations involving a common outcome, and two $\left(V_{1} A_{2}\right.$ and $\left.V_{2} A_{1}\right)$ allow the opportunity to observe summation of associations involving different outcomes.

\section{Method}

\section{Subjects and Apparatus}

The subjects were 16 experimentally naive male SpragueDawley rats about 90 days old. They were housed in individual cages and maintained on a food deprivation regime that kept them at $80 \%$ of their ad-lib body weight. They had free access to water in the home cage.

The apparatus consisted of eight operant chambers measuring $22.9 \times 20.3 \times 20.3 \mathrm{~cm}$, identical to those used in previous reports (e.g., Colwill \& Rescorla, 1985). The two end walls of each chamber were aluminum; the side walls and ceiling were clear Plexiglas. The floor of the chamber was composed of stainless steel rods with a $0.48-\mathrm{cm}$ diameter, spaced $1.9 \mathrm{~cm}$ apart, center to center. Each chamber had a recessed food magazine in the center of one end wall.

\begin{tabular}{l|ll} 
Train & \multicolumn{2}{|c}{ Test } \\
\hline$V_{1}-O_{1}$ & $V_{1}$ & $V_{1} A_{1}$ \\
$V_{2}-O_{2}$ & $V_{2}$ & $V_{2} A_{2}$ \\
$A_{1}-O_{1}$ & $A_{1}$ & $V_{1} A_{2}$ \\
$A_{2}-O_{2}$ & $A_{2}$ & $V_{2} A_{1}$
\end{tabular}

Figure 1. Design of Experiment 1. All the animals received Pavlovian conditioning with two auditory (A) and two visual (V) stimuli, each paired with one of two outcomes $(O)$. They were then tested with the conditioned elements and with all of the different-modality compounds. 
Two small metal cups measuring $1.25 \mathrm{~cm}$ in diameter and $1.5 \mathrm{~cm}$ deep were sunk side by side in the floor of each food magazine. An infrared detector and emitter system was mounted on the side walls of the magazine, permitting automatic recording of head movements into the magazine. To the left of the magazine was a lever, and to the right was a chain suspended from a microswitch mounted on the lid of the chamber. During Pavlovian conditioning procedures, access to these manipulanda was blocked by covering the lever with a metal shield and retracting the chain through a hole in the ceiling. Therefore, these manipulanda were not used in Experiment 1 but were used in related later experiments.

Each chamber was enclosed in a sound- and light-resistant shell. Mounted on the inside wall of this shell were speakers that permitted the presentation of a white noise $(\mathrm{N})$ and an $1800 \mathrm{~Hz}$ tone $(\mathrm{T})$, each measuring approximately $76 \mathrm{~dB}$ re $20 \mu \mathrm{N} / \mathrm{m}^{2}$ against a background level of $62 \mathrm{~dB}$ (C scale). Also mounted on that wall was a $6-W$ bulb that could be illuminated to provide a light $(\mathrm{L})$ stimulus during the otherwise dark session. Another 6-W light was mounted outside the rear wall of the chamber, at grid level; this light could be flashed at a rate of $1 / \mathrm{sec}$ to produce a flashing $(\mathrm{F})$ stimulus. The outside ceiling of the shell supported a solenoid-operated gravity feed valve that was connected via plastic tubing to the cups in the food magazine. This system permitted the presentation of $0.3 \mathrm{ml}$ of an $8 \%$ sucrose solution. Also attached to that food magazine was a dispenser containing 45-mg pellets (P. J. Noyes, Formula A).

Experimental events were controlled and recorded automatically by relays and microprocessors located in an adjoining room.

\section{Procedure}

Magazine training. On the first 2 days, the animals received a 20-min magazine training session, during which 20 noncontingent deliveries of pellets (Day 1) or sucrose (Day 2) were given, at time intervals variable around a mean of $1 \mathrm{~min}$.

Pavlovian conditioning. On each of the next 20 days, all animals received Pavlovian conditioning with four stimuli, L, F, T, and $\mathrm{N}$. Each session contained eight 30 -sec presentations of each stimulus, ending in an outcome. The contingencies were arranged so that, for half the animals, $\mathrm{L}$ was paired with pellets and $\mathrm{F}$ with sucrose, whereas for the other half of the animals, the outcomes were reversed. In a manner arranged to be orthogonal to those pairings, half the animals received $\mathrm{T}$ paired with pellets and $\mathrm{N}$ paired with su- crose, whereas half received the reversed contingencies. The intertrial interval (ITI), measured between stimulus initiations, was variable around a mean of 2 min, yielding a mean session duration of $64 \mathrm{~min}$. The number of head entries into the magazine was recorded during each 30 -sec stimulus and during the 30 -sec stimulus-free period preceding each stimulus.

Test. On the next day, all the animals received the first half of a standard conditioning session. This was followed without interruption by intermixed occurrences of two presentations each of the four elements and of the four cross-modal compounds (LN, LT, FN, and FT). The order of the test trials was balanced across stimulus and outcome identities. The elements continued to be reinforced with the appropriate outcomes; the compounds were nonreinforced. The ITI remained variable around a mean of $2 \mathrm{~min}$, yielding a session duration of $64 \mathrm{~min}$.

\section{Results and Discussion}

Conditioning proceeded without incident. On the final day, the mean response rates ranged from 18.8 to 22.2 magazine entries per minute during the stimuli, with no reliable differences as a function of stimuli or outcomes.

The data of most interest, from the test session, are shown in Figure 2. That figure separately displays responding prior to stimulus presentation, during the elements, during the compounds of elements signaling the same outcome, and during the compounds of elements signaling different outcomes. It is clear that each type of trial elevated responding relative to the prestimulus period but that the trial types differed among themselves. The compound of stimuli signaling a common outcome produced a higher level of responding than did the elements [Wilcoxon $T(16)=19, p<.01$ ]. Of more interest, the compound of the elements signaling different outcomes also elevated responding to a higher level than did the elements themselves $[T(16)=30, p<.05]$. In both cases, this comparison was made between the compound and that element that showed the stronger response for

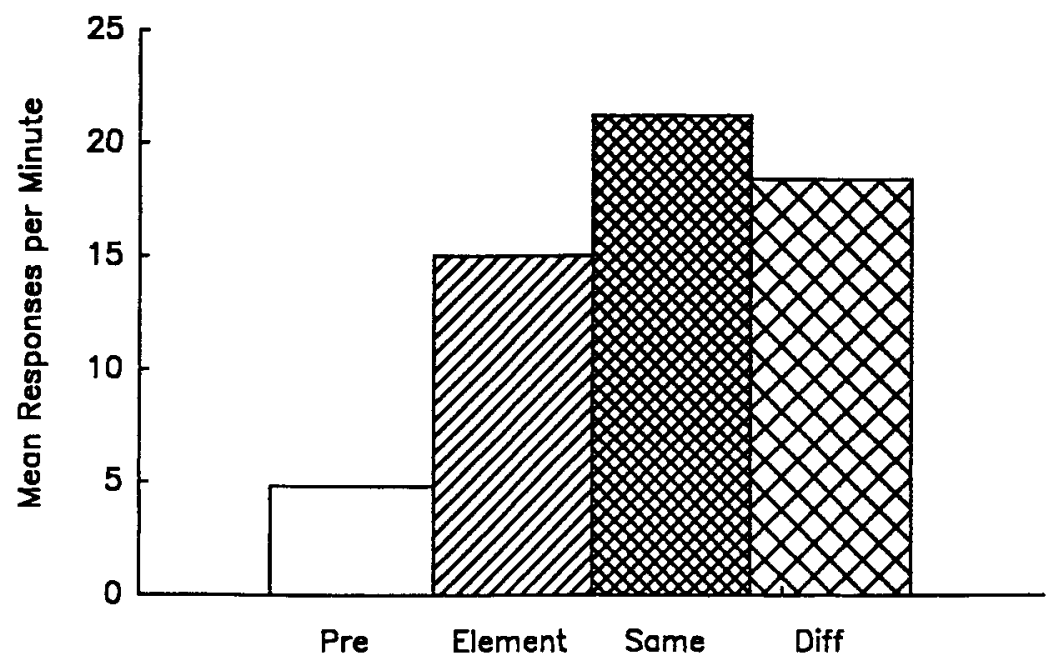

Figure 2. Test results of Experiment 1. Mean magazine entries are shown for responding prior to any stimulus presentation (pre), as well as during presentations of a stimulus element and of compounds consisting of elements signaling the same or different outcomes. 
each animal. As Rescorla (1997c) notes, this is a conservative comparison that avoids the possibility that summation is based on the animal's responding only to the stronger of the two components. Finally, the compound of elements signaling the same outcome produced a marginally higher response rate than did the compound of elements signaling different outcomes $[T(16)=31, .05<p<.10]$.

The pellet and sucrose outcomes produced similar levels of element performance during the test, confirming their similarity in value. The mean responses per minute were 14.2 and 16.2 for the auditory stimuli paired with pellets and sucrose, respectively. The comparable numbers for the visual stimuli paired with pellets and sucrose were 15.0 and 14.8. During the test, the standard errors of the mean (SEMs) ranged from a low of 2.3 to a high of 2.8 across the elements and compounds.

The finding of summation among stimuli signaling the same outcome confirms earlier observations with this preparation (e.g., Rescorla, 1997c). More interesting is the finding that summation also occurs among stimuli signaling different outcomes.

There have been several previous reports of substantial responding to compounds of elements signaling different outcomes in a magazine-approach preparation like that used here (Ganesan \& Pearce, 1988b; Watt \& Honey, 1997). Indeed, Watt and Honey found greater responding to compounds of elements signaling different outcomes than to compounds of elements signaling the same outcome. However, neither of those studies allowed evaluation of the greater responding to the compound than to the elements themselves, a comparison that is critical to the focus of the present report.

The importance of the present data is that they confirm summation, using the very same outcomes that have elsewhere failed to yield increased performance when interchanged in the course of training. These results support the view that such interchanging should have yielded enhanced performance, given that it preserves the integrity of associations with both outcomes.

The finding of marginally less summation for stimuli signaling different outcomes is also potentially of some interest. It supports the claim, based on differential devaluation and transfer experiments, that the present outcomes are discriminated by the animals. It may also be of analytic help in future investigations of the nature of summation itself. However, the recent report by Watt and Honey (1997) of greater summation with stimuli signaling different outcomes suggests that this difference may have little generality. In any case, for present purposes the central finding is that summation occurred when different outcomes were signaled by the stimuli presented in combination.

\section{EXPERIMENT 2A}

The finding of Experiment 1, that two stimuli paired with different outcomes show summation, raises the issue of why a single stimulus does not undergo an increase in responding when one of those outcomes is replaced by another. Because such a replacement results in a stimulus' having intact associations with both outcomes, one might have anticipated associative summation, resulting in substantial response augmentation. The failure to observe that augmentation (see, e.g., Rescorla, 1996a, 1998) leads one to search for some decremental process. One possibility is that, as the $\mathrm{S}-\mathrm{O}_{2}$ association grows, adding to the preexisting $\mathrm{S}-\mathrm{O}_{1}$ association, the total summed associative strength is discrepant from that which $\mathrm{O}_{2}$ can support. That "overexpectation" might produce a decrement in responding to the stimulus.

There is some evidence that such a decrement can occur when associations with the same outcome are combined. Beginning with the Rescorla-Wagner model (1972), many conditioning models have contained the assumption that associative change is governed by the discrepancy between the total outcome expected and that obtained. One consequence of such models is that a reinforcing outcome can produce decrement if it is inappropriately small, given the summed associative strength of the signaling elements. Early demonstrations of this result (e.g., Kamin \& Gaioni, 1974; Kremer, 1978; Rescorla, 1970; Wagner, 1971) employed aversive outcomes. But recently, Lattal and Nakajima (1998) have reported evidence for overexpectation in carefully designed experiments using the appetitive preparation employed here. They found that reinforcing a light-noise compound with the same outcome that had previously reinforced the light and noise separately led to decreases in performance to those elements. Moreover, Ganesan and Pearce (1988a) found evidence for summation and overexpectation with outcomes drawn from different motivational systems, water and pellets.

Consequently, the intention of Experiments $2 \mathrm{~A}$ and $2 \mathrm{~B}$ was to investigate whether such overexpectation could be at the heart of the decremental process observed in prior experiments from this laboratory when multiple outcomes are signaled. The design of Experiment 2A is illustrated in Figure 3. Following the procedures of Lattal and Nakajima (1998), these animals were initially trained with three stimuli, two of which were auditory and one

\begin{tabular}{l|r|l} 
Train & Overexpect & Test \\
\hline$V_{1}-O_{1}$ & $V_{1} A_{1}-O_{2}$ & \\
$A_{1}-O_{2}$ & $A_{2}-O_{2}$ & $A_{1}, A_{2}$ \\
$A_{2}-O_{2}$ & &
\end{tabular}

Figure 3. Design of Experiment 2A. All the animals received Pavlovian conditioning with one visual (V) and two auditory (A) stimuli, paired with one of two outcomes $(O)$. Then, one auditory stimulus was reinforced in compound with $V$, and the other presented alone. Both auditory stimuli were tested for their ability to elicit magazine responding. 
of which was visual. The two auditory stimuli signaled one outcome (either pellets or sucrose), and the visual stimulus signaled the other. The animals were then exposed to reinforced presentations of the compound of one of the auditory stimuli with the visual stimulus. In Experiment 2A, the outcome used for the compound was that which had been paired with the auditory elements; in Experiment 2B, it was that which had been paired with the visual element. It was anticipated that reinforcing the compound would result in overexpectation and, consequently, a depression in performance, whichever outcome was used.

In order to evaluate the degree of that depression, the other auditory stimulus was used as a comparison. In these experiments, that comparison stimulus received the same outcomes as did the compound during the compound phase. This allowed a comparison between two auditory stimuli, both of which were followed by the same outcome and only one of which was accompanied by the visual stimulus when it received that outcome. This comparison differs from that made by Lattal and Nakajima (1998), who omitted presentation of the comparison stimulus altogether during the compound phase. A different comparison was used here, for several reasons. First, it seemed useful to document overexpectation against an alternative but equally attractive comparison stimulus. Second, it allowed documentation of the occurrence of summation during the compound phase, by comparing responding to the compound with that to a counterbalanced auditory stimulus presented alone at the same point in the experiment. Third, it dealt with a concern, also noted by Lattal and Nakajima, that additional training of the element might, by itself, result in some decrement in performance unrelated to the occurrence of summation and overexpectation. In procedures using stimuli of this duration, additional training can result in inhibition of delay, evidenced by reduced responding early in the stimulus (see, e.g., Rescorla, 1997c). That reduction, which occurs even without the presence of another stimulus, could be mistaken for the decremental process that the present experiments were intended to assess. Consequently, it was useful to have a comparison stimulus that was also subjected to additional training and, therefore, to the possibility of the development of inhibition of delay.

\section{Method}

\section{Subjects and Apparatus}

The apparatus consisted of four chambers identical to those used in Experiment 1, with two exceptions. First, the light mounted on the outside rear wall of the chamber was moved to the center of the ceiling of the chamber; this light could be flashed at a rate of $1 / \mathrm{sec}$ to provide an $\mathrm{F}$ stimulus. Second, a relay mounted on the rear wall of the shell could be pulsed at a rate of $2 / \mathrm{sec}$ in order to provide a clicker $(C)$. The subjects were 16 rats of the same origin and maintained in the same manner as those in Experiment 1.

\section{Procedure \\ Magazine training. The animals received initial magazine train- ing in the manner of Experiment 1.}

Pavlovian conditioning. On each of the next 12 days, the animals received Pavlovian conditioning of N, C, and L. Each day contained eight 30-sec presentations of each stimulus with an ITI that was variable around a mean of $2.5 \mathrm{~min}$, yielding a session duration of $60 \mathrm{~min}$. For half of the animals, both auditory stimuli were terminated in a pellet, and $\mathrm{L}$ was terminated in a delivery of sucrose. For the other half of the animals, the outcomes were interchanged.

Compound treatment. Over the next 3 days, the animals received a treatment in which $\mathrm{L}$ was presented in compound with either $\mathrm{N}$ or $\mathrm{C}$ and terminated in one outcome. The other auditory stimulus was separately presented and terminated in that same outcome. The outcome used was the same as that used to train $\mathrm{N}$ and $\mathrm{C}$ during initial conditioning. The first compound conditioning session began with half of a Pavlovian conditioning session, delivering 4 presentations each of $\mathrm{L}, \mathrm{N}$, and $\mathrm{C}$. The session continued, without interruption, with eight $30-\mathrm{sec}$ presentations each of the compound and of the other auditory element, in intermixed order, each followed by the same outcome. The 2 nd day of compound training consisted of 16 presentations of each of these two kinds of trials. The 3rd day began with 8 of each trial type but then continued as a test session. Throughout this phase, the ITI (measured between stimulus initiations) was $2.5 \mathrm{~min}$

Test. There were two test sessions. The first was a continuation, without interruption, of the third compound session; the second was given on the next day. Each test session contained four nonreinforced 30-sec presentations each of $\mathrm{N}$ and $\mathrm{C}$, presented in counterbalanced order. The ITI remained $2.5 \mathrm{~min}$. The data of interest are the levels of performance to $\mathrm{N}$ and $\mathrm{C}$, as a function of the separate presentation or joint presentation with the light during the compound phase.

\section{Results and Discussion}

Initial acquisition proceeded smoothly. The mean rates of magazine entry on the final conditioning day were 17.6, 16.4 , and 5.8 per minute, for the auditory stimuli, the visual stimulus, and the prestimulus period, respectively.

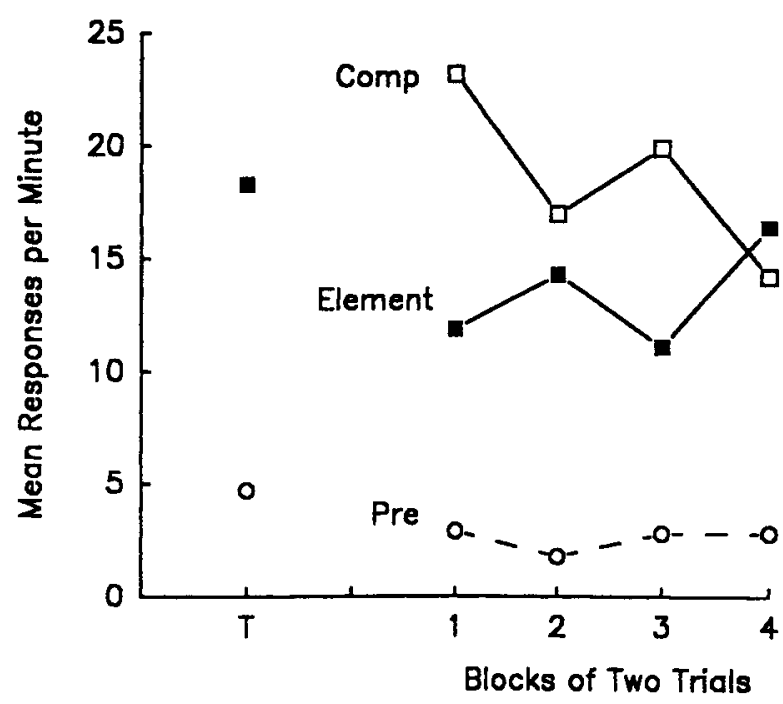

Figure 4. Results of the initial compound conditioning trials in Experiment 2A. Responding is shown prior to any stimulus presentation (pre), during the one auditory element, and during the compound of the other auditory element and the visual stimulus. The points to the left show responding in the prestimulus period and during the auditory stimuli in the first half of the session. 
The data points to the left in Figure 4 show the response rates for the auditory stimuli in the first half of the first compound session, when simple conditioning of the elements continued. During that period, the mean rate of magazine entry during the light was 18.6 per minute.

The main portion of Figure 4 shows responding during the second half of the first compound session, when one auditory stimulus continued to be presented alone and the other was presented in compound with the light. On the first two trials of that sort, there was substantially more responding to the compound than to the auditory element alone $[T(15)=6, p<.01]$, suggesting that summation occurred in the compound stimulus. On these trials, the SEMs were 2.1 and 1.4 for the compound and element, respectively. It is worth noting that this different level of responding was partly attributable to a disruption of the responding to the element presented alone during the compound phase of the experiment. Consequently, as Lattal and Nakajima (1998) speculated, summation is best observed in terms of a comparison of responding to the compound and to the element during the same phase of the experiment, rather than in terms of a comparison between phases. In any case, this confirms the observation of Experiment 1, that there is summation when stimuli signaling different outcomes are combined. However, over the course of this session, responding converged for the two trial types and then remained similar throughout the rest of compound training. On the final four trials of compound training, at the beginning of the test session, the mean response rates were 18.0 and 18.8 per minute, for the compound and auditory element, respectively.

The data of primary interest, from the test of the auditory stimuli presented alone, are shown in Figure 5, separated for the 2 days of testing. That figure shows respond- ing separately in the prestimulus period, during the auditory stimulus that had been presented in compound with the light, and during the auditory stimulus that had been presented alone. To the left are shown the terminal rates of responding to those stimuli on the final day of element training. Although both stimuli continued to elevate responding relative to that observed in the prestimulus period throughout the test, the elevation was substantially less for the auditory stimulus that had been reinforced in compound with the light. Over the entire test period, that difference proved reliable $[T(16)=19, p<.01]$. Over that test, the SEMs were 2.1 and 1.6 for the stimulus subjected to overexpectation and the control stimulus. These results suggest that the consequence of reinforcing the stimulus in compound with a stimulus signaling another outcome was to reduce performance.

These results support the notion that the summation in performance observed when stimuli signaling different outcomes are combined leads to an overexpectation of the outcome. The result of pairing that compound with the outcome previously used to train the auditory stimulus was a decrement in subsequent performance to that auditory stimulus. That is, overexpectation was observed with stimuli signaling different outcomes.

\section{EXPERIMENT 2B}

The results of Experiment $2 \mathrm{~A}$ suggest that, when a compound of elements conditioned by $\mathrm{O}_{1}$ and $\mathrm{O}_{2}$ is followed by only one of those outcomes $\left(\mathrm{O}_{2}\right)$, there will be a decrease in the subsequent performance of that element that previously signaled that outcome $\left(\mathrm{O}_{2}\right)$. This conclusion was based on a comparison with a comparable element that received continued training with that same $\mathrm{O}_{2}$.

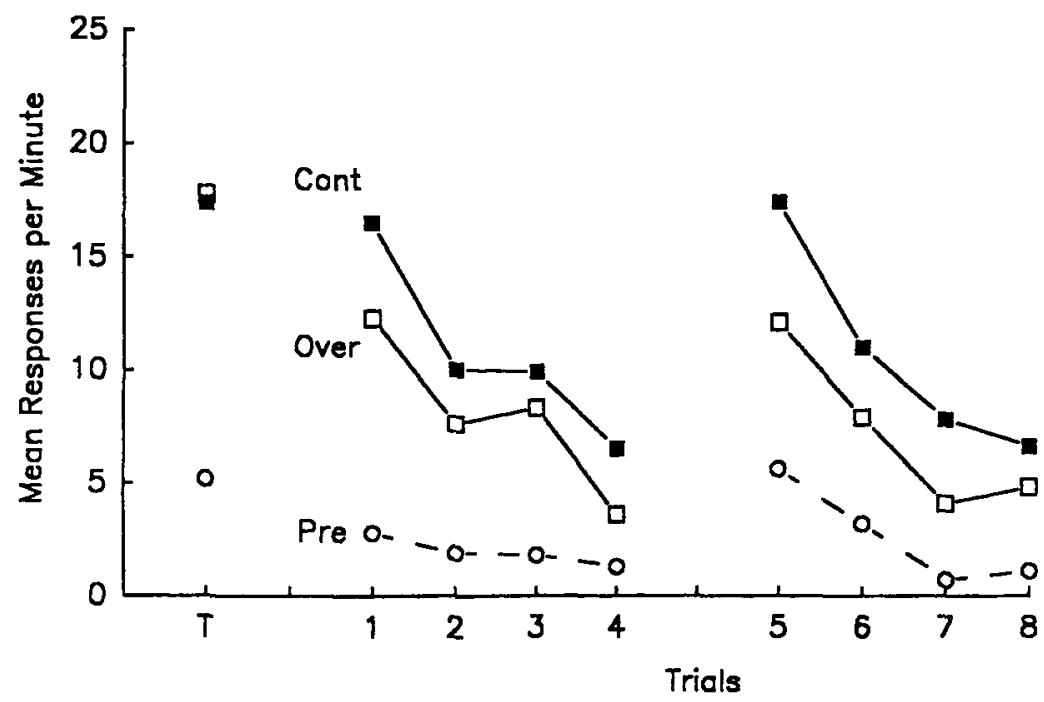

Figure 5. Test results from Experiment 2A. Responding is shown over the course of the two test sessions for the auditory stimulus subjected to overexpectation training and the control auditory stimulus, as well as during the prestimulus period. The points to the left show performance to those auditory stimuli on the last day of their conditioning prior to compound conditioning. 
However, this design did not allow for a comparison of the effects of reinforcing the compound with $\mathrm{O}_{2}$ on the other element, whose conditioning history had been with $\mathrm{O}_{1}$. It is of some theoretical interest to know whether reinforcing the compound with $\mathrm{O}_{2}$ results in a decrement both in the stimulus with a history of pairing with $\mathrm{O}_{2}$ and in that with a history of pairing with $\mathrm{O}_{1}$. Consequently, in Experiment 2B, the outcome used following the compound was that which had previously reinforced the visual stimulus. As in Experiment 2A, a comparison auditory stimulus received comparable initial conditioning but then was reinforced alone. One can then assess the depressive effect of reinforcing the compound with $\mathrm{O}_{1}$ on a stimulus with a history of conditioning by $\mathrm{O}_{2}$.

\section{Method}

The procedure of Experiment 2B was identical to that of Experiment $2 \mathrm{~A}$, except that the outcome used during the compound phase of the experiment was that previously signaled by the light rather than by the auditory stimuli.

\section{Results and Discussion}

Despite the fact that the same initial conditioning procedures were used as those in Experiment 2A, the overall level of responding was generally somewhat lower. This level of variation is not uncommon for this laboratory across different shipments of animals. Although the overall pattern of results was similar to that of Experiment $2 \mathrm{~B}$, this suggests added caution in making crossexperiment comparisons.

On the final day of conditioning, the mean response rates during the auditory, visual, and prestimulus periods were $13.1,10.8$, and 2.6 per minute, respectively.

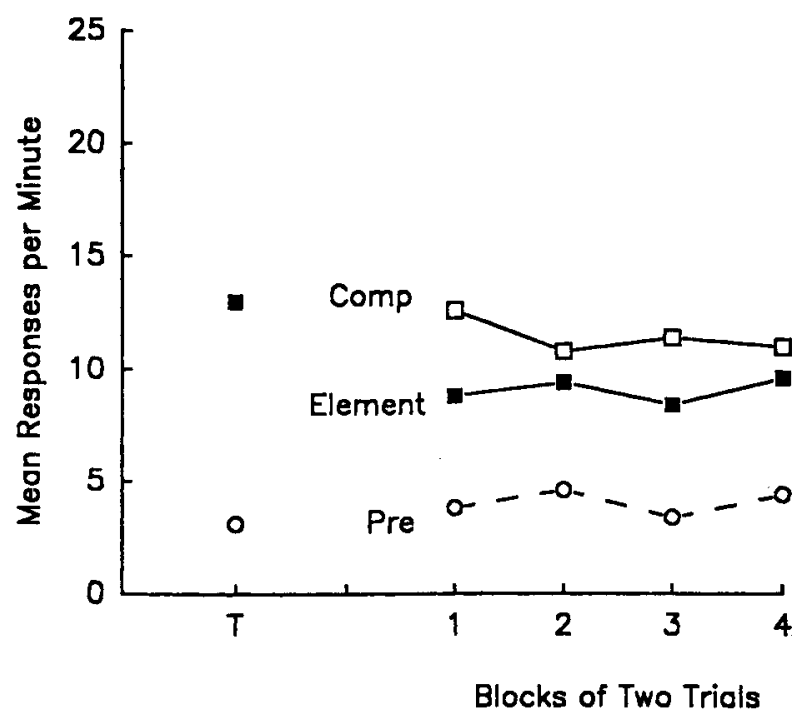

Figure 6. Results of the initial compound conditioning trials in Experiment 2B. Responding is shown prior to any stimulus presentation (pre), during the one auditory element, and during the compound of the other auditory element and the visual stimulus. The points to the left show responding in the prestimulus period and during the auditory stimuli in the first half of the session.
The left portion of Figure 6 displays responding to the auditory stimuli in the first half of the first compound session. During that period, the mean response rate during the light was 10.2 responses per minute. The main portion of Figure 6 shows responding during the compound conditioning phase separately for the compound, the auditory element alone, and the prestimulus period. As in Experiment 2A, responding was initially greater during the compound than with the auditory stimulus alone $[T(16)=24, p<.02 ; S E M \mathrm{~s}=1.7$ and 1.5], but the two rates converged during the session. Responding to the compound and the auditory element remained similar throughout the rest of compound training. On the final four such trials, in the first half of the test session, the mean responses per minute were 13.2 and 14.1, respectively, for the compound and the auditory element.

The results from the test of the auditory stimuli alone are shown in Figure 7. To the left are the terminal levels of responding on the last day of element conditioning. As in Experiment 2A, throughout the test there was more responding during the control stimulus than during that which had been subjected to reinforcement in compound with the light. Over the two test sessions, that difference proved reliable $[T(16)=29, p<.05 ; S E M \mathrm{~s}=1.8$ and 1.2]. This pattern of results is quite similar to that of Experiment $2 \mathrm{~A}$.

Together, Experiments $2 \mathrm{~A}$ and $2 \mathrm{~B}$ suggest that there is a decrement in performance to an auditory stimulus reinforced in compound with the light, whether the outcome following the compound is that previously used to train the light or the auditory stimulus. That, in turn, suggests that whichever outcome is used, both stimuli undergo performance decrement. This supports the second claim made in the introduction - that the summation that arises from stimuli signaling different outcomes results in an overexpectation that leads to performance decrement. These results agree with those of Ganesan and Pearce (1988b), who found evidence for reduction in performance when stimuli signaling food and water were presented in compound and then followed by one of those outcomes.

\section{EXPERIMENT 3A}

The intention of Experiments $3 \mathrm{~A}$ and $3 \mathrm{~B}$ was to investigate in more detail the nature of the performance decrement resulting from an overexpectation procedure. According to the argument developed here, that decrement does not involve a change in the associations with the outcomes. Rather, some outcome-independent depressive process is superimposed on the $\mathrm{S}-\mathrm{O}_{1}$ and $\mathrm{S}-\mathrm{O}_{2}$ associations, masking their summation in performance. The soundness of that argument cannot be evaluated simply by looking at overall performance to $\mathrm{S}$. Rather, some procedure must be adopted that allows assessment of the state of the $\mathrm{S}-\mathrm{O}_{1}$ and $\mathrm{S}-\mathrm{O}_{2}$ associations with and without an overexpectation procedure.

In these experiments, the state of those $\mathrm{S}-\mathrm{O}$ associations was assessed by a transfer procedure, in which the 


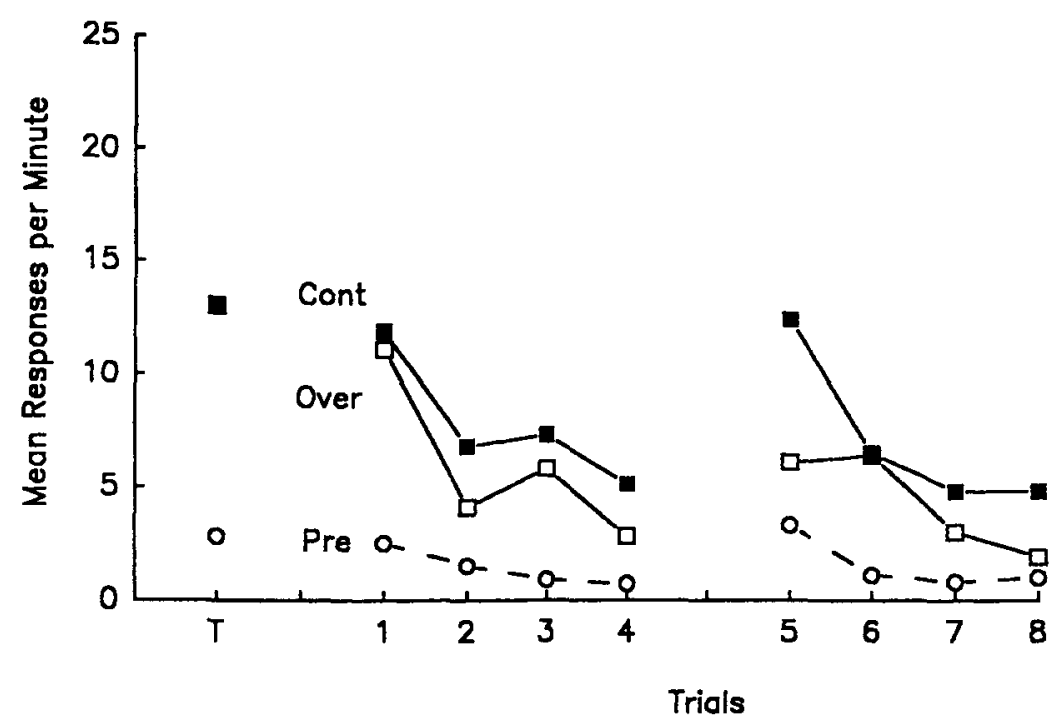

Figure 7. Test results from Experiment 2B. Responding is shown over the course of the two test sessions for the auditory stimulus subjected to overexpectation training and the control auditory stimulus, as well as during the prestimulus period. The points to the left show performance to those auditory stimuli on the last day of their conditioning prior to compound conditioning.

stimuli were superimposed on instrumental responses that had a history of earning the different outcomes. It is routine to observe that a stimulus having a Pavlovian association with an outcome selectively encourages responses that have earned that same outcome (see, e.g., Colwill \& Rescorla, 1988). Consequently, in these experiments, several auditory stimuli received Pavlovian conditioning, then one stimulus was subjected to an overexpectation procedure, and finally both were tested for their ability to augment responses with which they shared an outcome. Experiment $3 \mathrm{~A}$ examined the effects of overexpectation

\begin{tabular}{|c|c|c|c|c|}
\hline $\begin{array}{c}\text { Response } \\
\text { Training }\end{array}$ & Condit & Overexp & & Test \\
\hline & $A_{1}-O_{1}$ & & \multirow{4}{*}{\multicolumn{2}{|c|}{$\begin{array}{l}A_{1} \\
A_{2}\end{array}$}} \\
\hline$R_{1}-O_{1}$ & $A_{2}-O_{2}$ & $V_{1} A_{1}-o_{1}$ & & \\
\hline $\mathrm{R}_{2}-\mathrm{O}_{2}$ & $v_{1}-o_{1}$ & $\mathrm{~A}_{2}-\mathrm{O}_{2}$ & & \\
\hline & $v_{2}-o_{2}$ & & & \\
\hline
\end{tabular}

Figure 8. Design of Experiment 3A. Initially, animals were given instrumental training with two responses $\left(R_{1}\right.$ and $\left.R_{2}\right)$, each resulting in a different outcome $\left(O_{1}\right.$ or $\left.O_{2}\right)$. Then they received Pavlovian conditioning with two auditory (A) and two visual (V) stimuli, using different outcomes. During the next phase, one auditory stimulus was presented in compound with a visual stimulus and the other presented alone, both followed by their original outcomes. In the test, the two auditory stimuli were presented at a time when the animal had both $R_{1}$ and $R_{2}$ available. when both stimuli of the compound had been paired with the same outcome. Experiment 3B repeated the procedure for stimuli that had signaled different outcomes.

The design of Experiment 3A is sketched in Figure 8. Initially, two different responses $\left(R_{1}\right.$ and $R_{2}$, a leverpress and a chain pull) were reinforced by two different outcomes, $\mathrm{O}_{1}$ and $\mathrm{O}_{2}$, counterbalanced as pellets and sucrose. Then Pavlovian conditioning was conducted with two auditory stimuli $\left(\mathrm{A}_{1}\right.$ and $\mathrm{A}_{2}$, counterbalanced as noise and clicker) and two visual stimuli $\left(V_{1}\right.$ and $V_{2}$, counterbalanced as flashing light and houselight). Within each modality, one stimulus was followed by $\mathrm{O}_{1}$ and one by $\mathrm{O}_{2}$. In the final test, the auditory $\mathrm{A}_{1}$ and $\mathrm{A}_{2}$ stimuli were presented while the animal had a choice between making $R_{1}$ or $R_{2}$. The expectation was that, in the absence of any other treatment, $A_{1}$ would selectively encourage responding to $R_{1}$, whereas $A_{2}$ would selectively encourage responding to $R_{2}$. However, prior to the test, one of the auditory stimuli, $A_{1}$, was subjected to an overexpectation treatment involving its reinforcement in conjunction with $V_{1}$, whereas the other auditory stimulus, $A_{2}$, continued to be reinforced alone as in initial conditioning.

Two other features of this experiment, both intended to maximize the sensitivity of the transfer test, should be noted. First, unlike Experiments $2 \mathrm{~A}$ and 2B, the overexpectation treatment used here involved comparing two auditory stimuli that had histories of signaling different outcomes. This ensures that, during the test, each instrumental response will be more augmented by one of the two stimuli and, hence, minimizes the development of an overall response bias during the test. Second, the test of transfer was conducted immediately after the last com- 
pound trial, without a separate intervening test of the simple Pavlovian response to the auditory stimuli. However, in order to verify the successful depression of the Pavlovian response, magazine entries were also recorded during the test of transfer to leverpressing and chain pulling.

\section{Method}

\section{Subjects and Apparatus}

The subjects were 16 male rats of the same type and maintained in the same manner as those in previous experiments. The apparatus was that of Experiment 1, with the ability to present a clicker added. In appropriate phases, the lever and chain were made available.

\section{Procedure}

Response training. On each of the first 2 days, the animals received a 20 -min magazine training session with pellets and sucrose, respectively. Each session contained 20 deliveries of one outcome. Over the next 2 days, all the animals were trained in separate sessions to leverpress and chain pull. Each training session allowed responding to earn 25 deliveries of an outcome on a continuous reinforcement schedule. For half the animals, leverpressing led to a pellet and chain pulling to sucrose; for the other half of the animals, the $\mathrm{R}-\mathrm{O}$ relations were interchanged. On each of the next 5 days, all the animals received VI training with the lever and chain. Each day contained two 20-min sessions, one with lever and one with chain, spaced about an hour apart. During each session, responding was reinforced on a variable interval (VI) 1 -min schedule, using the reinforcer employed during initial training.

Pavlovian conditioning. On the next 12 days, the animals received Pavlovian conditioning of $\mathrm{N}, \mathrm{C}, \mathrm{L}$, and $\mathrm{F}$, in the manner of prior experiments. Each trial was $30 \mathrm{sec}$ long and terminated in an outcome. Each session contained eight presentations of each of the four stimuli, counterbalanced with regard to the outcome delivered, under the constraint that $\mathrm{N}$ and $\mathrm{C}$ were paired with different outcomes; $\mathrm{L}$ and $\mathrm{F}$ were also paired with different outcomes, in a manner orthogonal to that for $\mathrm{N}$ and $\mathrm{C}$. The lever and chain were absent during these sessions. Throughout the remainder of the experiment, the mean ITI was $2.5 \mathrm{~min}$. This yielded 80 -min conditioning sessions.
Transfer test preparation. Between Days 11 and 12 of Pavlovian conditioning, the animals received 2 retraining days on the instrumental responses. On the first of these, they received two VI sessions in the manner of prior training. On the second, they received an 8-min choice session on which both lever and chain were present but not rewarded. Prior work has shown such sessions to equalize the rates of the two responses and enhance sensitivity to transfer.

Compound conditioning. On the next 3 days, the animals received a compound conditioning treatment like that used in Experiment $2 \mathrm{~A}$. The first compound conditioning session was the same as the first half of a Pavlovian conditioning session, delivering 4 presentations each of L, F, N, and C. It continued without interruption, with 8 presentations of the compound and of the other auditory element, in intermixed order, with each auditory stimulus followed by the same outcome as it had received during initial conditioning. The 2 nd day of compound training consisted of 16 presentations of each of these two kinds of trials. Both of these sessions were $80 \mathrm{~min}$ long. The 3 rd day began with 8 presentations of each trial type, yielding a 40-min conditioning session, but then continued as a test session. The lever and chain were absent during compound conditioning sessions.

Test. Immediately after the third session of compound conditioning, the animals were removed from the chambers, and the lever and chain uncovered. The animals were then returned to the chambers and received four 30 -sec presentations each of $\mathrm{N}$ and $\mathrm{C}$, in counterbalanced order, delivered with an interstimulus interval of $30 \mathrm{sec}$, producing an 8-min session. The results of primary interest are the rates of responding on these manipulanda during $\mathrm{N}$ and $\mathrm{C}$, as a function of which stimulus had been subjected to an overexpectation treatment. In addition, the rate of magazine responding was recorded during the $30-\mathrm{sec}$ prestimulus and stimulus periods.

\section{Results and Discussion}

Initial instrumental response and Pavlovian conditioning proceeded as expected. By the final day of VI training, the rate of making the instrumental response was 9.6 per minute, with no reliable differences as a function of the identity of the manipulandum or the outcome. Ter-

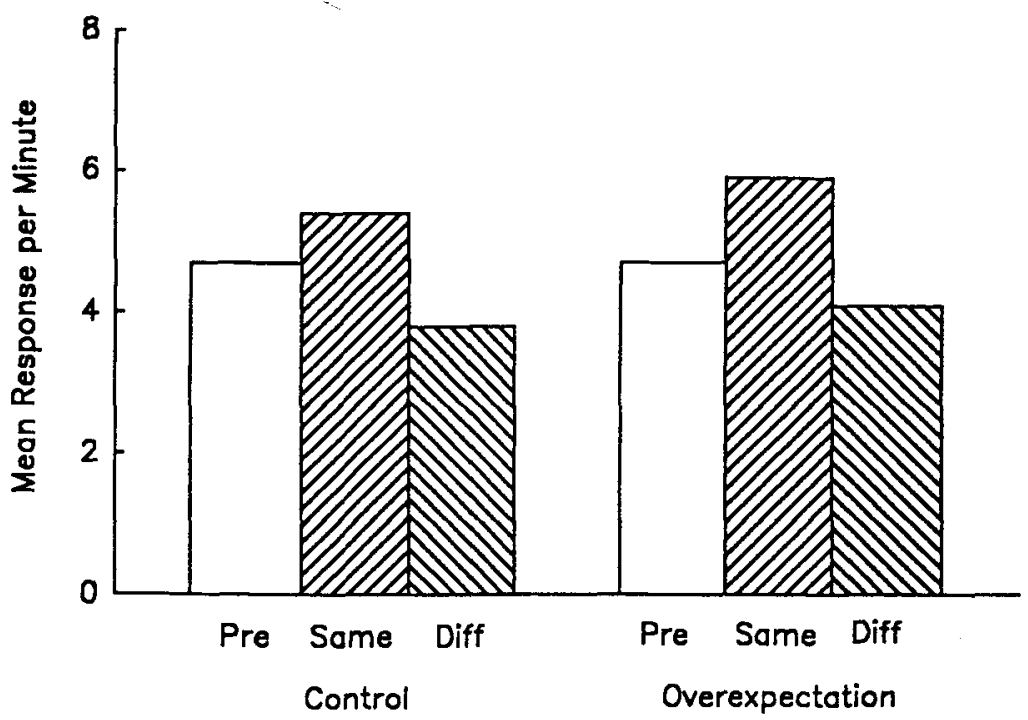

Figure 9. Test results from Experiment 3A. Responding is shown for a control stimulus and one subjected to overexpectation on manipulanda that had earned the same or a different outcome from that with which the stimulus was conditioned. Responding is also shown for the prestimulus period. 
minal levels of responding on the two auditory and two visual stimuli were 12.9 and 11.9 , respectively.

Summation was observed on the 1st day of compound training. On that day, the mean magazine entries per min were 15.3 and $11.7,(S E M \mathrm{~s}=0.99$ and 0.84$)$, respectively, for the compound and separately presented element $[T(15)=11, p<.01]$. However, by the final day of compound training, the response levels had converged somewhat to 14.5 and 13.4 responses per minute, respectively.

The data of most interest, from the transfer test when the auditory stimuli were superimposed on the responses, are shown in Figure 9. That figure shows the rates of responding prior to a stimulus presentation, during a stimulus that signaled the same outcome as the response had earned, and during a stimulus that signaled a different outcome from that which the response had earned. The data are displayed separately for the auditory stimulus that had been subjected to overexpectation and that which was a control. The data pattern for the control stimulus is familiar from earlier reports (e.g., Colwill \& Rescorla, 1988). Presenting a Pavlovian stimulus better promoted the response that had earned the same outcome than that which had earned a different outcome. As in earlier reports, the effect was evident primarily as differential depression relative to the prestimulus response rate.

Of particular interest, essentially the identical data pattern was observed with the stimulus subjected to overexpectation. There were no reliable differences between the two stimuli, but together they reliably augmented the same-outcome response more substantially $[T(14)=9.5$, $p<.01]$. During this test, the $S E M$ s ranged from 0.92 to 1.01. These results suggest that subjecting a stimulus to an overexpectation treatment had little detrimental effect on its ability to transfer on the basis of the outcome with which it had been paired. That suggests that the $\mathrm{S}-\mathrm{O}$ association persisted largely intact through the overexpectation treatment.

At the same time that the stimulus continued in its ability to evoke outcome-based transfer, however, it was diminished in its ability to evoke the original Pavlovian response. During the test, the mean rates of magazine entry were 8.5 and $12.5(S E M \mathrm{~s}=1.4,2.0)$ for the stimulus subjected to overexpectation and the control stimulus, respectively $[T(16)=22.5, p<.02]$. That is, overexpectation, in the sense of decreased ability to evoke the original response, was observed here, as in the work of Lattal and Nakajima (1998).

These results suggest that the decrement observed in a standard overexpectation experiment, using a single outcome, leaves the original $\mathrm{S}-\mathrm{O}$ associations intact. Experiment $3 \mathrm{~B}$ examines whether this is also the case when multiple outcomes are used.

\section{EXPERIMENT 3B}

This experiment was identical to Experiment $3 \mathrm{~A}$, with one exception. The outcome identities were interchanged for the visual stimuli, so that $\mathrm{V}_{1}$ was paired with $\mathrm{O}_{2}$ and $\mathrm{V}_{2}$ with $\mathrm{O}_{1}$. The consequence of this change is that, during the overexpectation phase of the experiment, the VA compound contained elements that had been conditioned with different outcomes. As in Experiment $3 \mathrm{~A}$, the outcome that followed that compound was the same as the outcome previously signaled by the auditory stimulus alone. The question of interest is whether the depression in magazine performance anticipated to the auditory stimulus, on the basis of the results of Experiments $2 \mathrm{~A}$ and $2 \mathrm{~B}$, occurred despite the preservation of the original

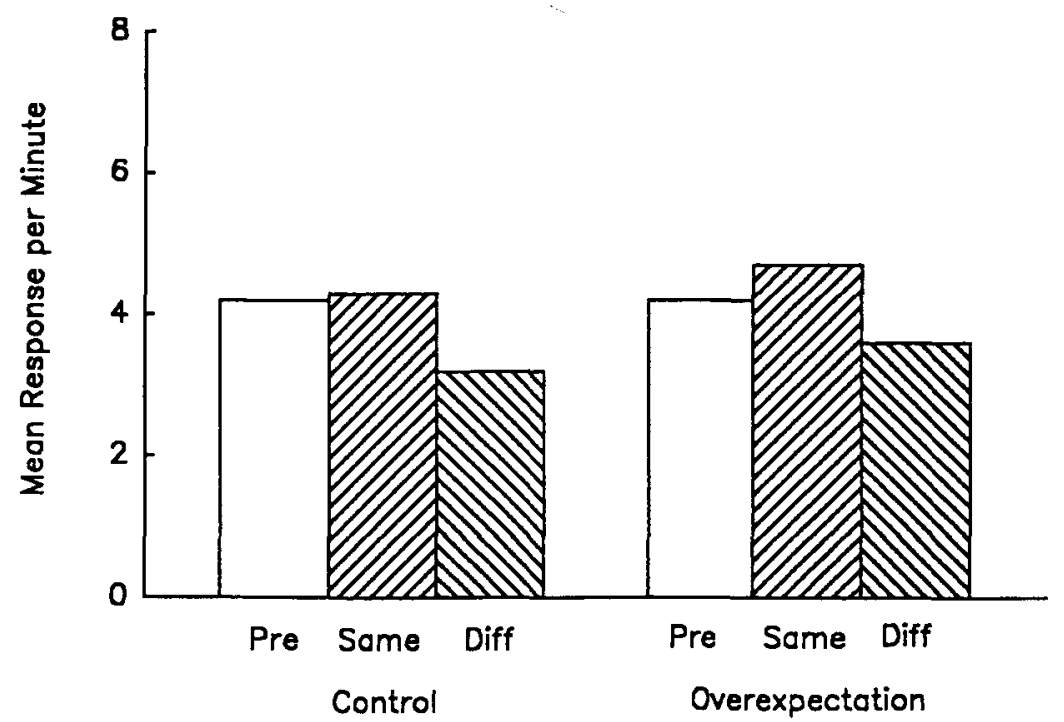

Figure 10. Test results from Experiment 3B. Responding is shown for a control stimulus and one subjected to overexpectation on manipulanda that had earned the same or a different outcome from that with which the stimulus was conditioned. Responding is also shown for the prestimulus period. 
outcome associations, as assessed in the transfer test. That is, will the overexpectation result produced by using different outcomes leave the original outcome associations intact in the way that overexpectation produced by a common outcome did in Experiment $3 \mathrm{~A}$ ?

\section{Method}

\section{Subjects and Apparatus}

The subjects were 16 male rats of the same type and maintained in the same manner as those in previous experiments. The apparatus was that of Experiment $3 \mathrm{~A}$.

\section{Procedure}

The procedure was identical to that of Experiment $3 \mathrm{~A}$, except for the interchanging of the reinforcer identities for the visual stimuli during the initial conditioning phase.

\section{Results and Discussion}

The results were very similar to those of Experiment $3 \mathrm{~A}$. Pavlovian conditioning and instrumental training developed without incident. On the final day of VI training, the rate of instrumental responding was 9.6 per minute. Terminal levels of responding on the two auditory and two visual stimuli were 13.3 and 15.1 , respectively. Summation occurred on the 1st day of compound training; the mean magazine entries were 18.8 and 11.4 (SEMs = 1.21 and 1.03) for the compound and element, respectively $[T(16)=18, p<.01]$. By the final day of compound training, the levels had converged somewhat to 16.1 and 13.8 responses per minute, respectively.

Figure 10 shows the results of the transfer test, during which the treated and control stimuli were presented while the lever and chain were available. The data pattern observed during the test was quite like that of Experiment $3 \mathrm{~A}$. For both the control stimulus and the treated stimulus, responding was greater on the same-outcome response than on the different-outcome response. There were no reliable differences in performance between the two stimuli, but together they produced reliably more responding on the same-outcome response $[T(16)=28$, $p<.05 ; S E M \mathrm{~s}=0.56$ and 0.81$]$.

During the test, the treated stimulus showed a decrement in its ability to evoke the Pavlovian magazine response, as compared with the control stimulus. The mean magazine entries were 7.3 and $9.4(S E M s=1.0$ and 1.2) per minute for the stimulus subjected to overexpectation and the control stimulus, respectively $[T(16)=29, p<.05]$.

There results confirm, for the case of two outcomes, the results of Experiment 3A. Although the overexpectation procedure produced a decrement in performance of the original response, it left substantial $\mathrm{S}-\mathrm{O}$ associations, as measured by transfer. Of course, caution must always be exercised in interpreting the absence of differences between treatments. One cannot be certain that there was no negative impact on the $\mathrm{S}-\mathrm{O}$ associations. However, the present procedures were sufficiently powerful to detect the impact of compound conditioning on mag- azine approach as well as the selective control of Pavlovian stimuli on instrumental responses with which they shared an outcome. In any case, the essential finding is that stimuli subjected to overexpectation continue to show excellent transfer, suggesting that the substantial $\mathrm{S}-\mathrm{O}$ associations survived the overexpectation treatment.

\section{GENERAL DISCUSSION}

The present results demonstrate summation of associations with discriminably different outcomes of the same affective value. They also show that this summation is sufficiently large to evoke responding greater than that which would be supported by one of the outcomes and, therefore, to produce overexpectation when only one outcome is presented. Finally, they suggest that this overexpectation acts to leave intact the original $\mathrm{S}-\mathrm{O}$ associations but to superimpose an outcome-independent depressive process.

This pattern of findings helps make sense out of a variety of results from experiments in which one outcome is substituted for another in the course of conditioning. The observation that such a shift among equally valued outcomes produces little change in responding while concurrently increasing the number of outcome-based associations can be accommodated as a case of outcomeindependent response depression based on the summation and resultant overexpectation of the two disparate outcomebased associations.

The summation results of Experiment 1 are in agreement with several prior reports that combined conditioned stimuli (CSs) signaling different outcomes. Ganesan and Pearce (1988b) and Watt and Honey (1997) have both reported substantial magazine responding to an $A B$ compound when the $A$ and $B$ elements had previously signaled different outcomes. Although neither of those reports allowed a comparison with responding to the elements themselves, taken together with the present results, they strongly support the view that summated responding should occur across CSs signaling equivalently valued outcomes. The special virtue of the present Experiment 1 is that it demonstrates summation across outcomes known from earlier experiments in this laboratory to be easily discriminable, similarly valued, and both fully encoded when a single stimulus signals them successively. This combination of maintained encoding of outcomes that otherwise summate is what necessitates the analysis conducted in Experiments 2 and 3.

The overexpectation results of Experiments $2 \mathrm{~A}$ and 2B have also been foreshadowed by Ganesan and Pearce (1988b). Using a between-subjects design, they found that reinforcing an $\mathrm{AB}$ compound with either food or water resulted in loss of responding both to an A that had signaled water alone and to $\mathrm{A}$ that had signaled food alone. Indeed, they concluded that there was no reason to believe that the animals discriminated the different outcomes 
in their study, a possibility that was especially encouraged by their using a common auditory stimulus in conjunction with the delivery of both outcomes.

The results of the present Experiments $3 \mathrm{~A}$ and $3 \mathrm{~B}$ are of particular interest because they provide information about the detailed associative changes that occur in overexpectation. The original predictions of overexpectation were derived from the Rescorla-Wagner (1972) model, according to which increases or decreases in the associative strength of a stimulus are based on the discrepancy between the current strength and that which the outcome will support. According to a model of that type, a stimulus will undergo loss in its associative strength, despite its being reinforced, if it is presented in a compound whose current strength is larger than that which the reinforcer will support. That is, overexpectation should result in a reduction in the originally learned associations. However, the results of the present experiments suggest that this interpretation may be incorrect; overexpectation may produce a behavioral decrement that does not have its origin in the reduction of the original associations. In this regard, the present experiments highlight a difficulty for such models that has frequently been pointed out for the case of extinction: Processes that produce behavioral decrements may not represent reductions in the underlying associations but rather the superimposition of some inhibitory process.

The nature of that inhibitory process is not entirely clear. However, one interpretive possibility would be to follow a suggestion made by Konorski (1967) and developed in more detail by Wagner (e.g., Betts, Brandon, \& Wagner, 1996). Konorski argued that outcomes should be conceptualized as having some unique and some shared features, in the matter in which signals are routinely described. In that case, one could think of pellets and sucrose as having distinctive sensory components but sharing affective and response components. Compounds of stimuli that have signaled the different outcomes would lead to summation based in part on those shared components. Moreover, should that compound be reinforced by one of the outcomes, the shared components would be inappropriately large, providing occasion for their decrement. For instance, affective decrements might take the form of superimposing a negative affect while allowing preservation of the original learning. The overexpectation observed here and in extinction might lead to frustration, which could become conditioned to the stimuli (see, e.g., Amsel, 1958, 1967).

The envisioning of a frustration process being conditioned to the stimuli would have a number of theoretical advantages. By counteracting the positive affect of the outcomes, it would act to limit the total affective reaction. However, to the degree that it depends only on the affective nature of the outcomes, it would not specifically undermine the sensory information but, instead, leave intact information about the sensory features of the outcomes. As a result, the conditioning of frustration might limit the abil- ity of the stimuli to motivate their original behaviors, while allowing the occurrence of transfer based on sensory properties. Moreover, it could provide a mechanism for changes in performance with time, such as spontaneous recovery from extinction or from the decrements involved in replacing one outcome by another, if the separate frustration process were partially to dissipate with time. Finally, frustration might also be conditioned to the particular responses that are evoked when it occurs, providing a means to account for the high degree of response specificity reported in earlier experiments (e.g., Rescorla, 1995).

Summation and overexpectation are two of several compound stimulus procedures important to contemporary theories of conditioning. It is, therefore, of interest to inquire about the possible implications of the present results for other instances in which stimuli are presented in compound. One especially important case is that of blocking, in which an $\mathrm{AB}$ compound is followed by an outcome after prior conditioning of $\mathrm{A}$ alone (see, e.g., Kamin, 1968). It is routine to find that such prior conditioning of A sharply attenuates (blocks) the conditioning of $B$ that would otherwise result from the AB trials. Although this blocking can sometimes be disrupted by changes in the intensity or frequency of the outcome (see, e.g., Dickinson, Hall, \& Mackintosh, 1976; Holland, 1984), there have been several reports that blocking can persist, even under circumstances in which the identity of the outcome following $\mathrm{AB}$ is different from that following A alone (e.g., Bakal, Johnson, \& Rescorla, 1974; Ganesan \& Pearce, 1988a; Williams, 1994). One possible account of this blocking under changes in outcome identity would exploit the current findings on overexpectation. Even if changing the identity of the outcome in a blocking experiment allowed B to develop an association with the new outcome, the resulting summation of the associations of $A$ and $B$ with their different outcomes would result in overexpectation. When the $A B$ compound is then reinforced, this could result in the kind of outcome-independent behavioral decrement observed in the present overexpectation experiments. The result would be the appearance that new learning had been blocked despite both the development of new S-O associations and the resulting development of an outcomeindependent decremental process. Clearly, a more detailed analysis of the $\mathrm{S}-\mathrm{O}$ associations would be needed to settle that question.

In any case, the present experiments suggest that summation and overexpectation function across different outcomes in a way that has important implications for our understanding of associative learning.

\section{REFERENCES}

AMSEL, A. (1958). The role of frustrative nonreward in noncontinuous reward situations. Psychological Bulletin, 55, 102-119.

AMSEL, A. (1967). Partial reinforcement effects on vigor and persistence. In K. W. Spence \& J. T. Spence (Eds.), The pswchologr of learning and motivation (Vol. 1. pp. 1-65). New York: Academic Press. 
Bakal, C. W., Johnson, R. D., \& Rescorla, R. A. (1974). The effect of change in US quality on the blocking effect. Pavlovian Journal of Biological Science, 9, 97-103.

BetTs, S. L., Brandon, S. E., \& Wagner, A. R. (1996). Dissociation of the blocking of conditioned eyeblink and conditioned fear following a shift in US locus. Animal Learning \& Behavior, 24, 459-470.

Colwill, R. M., \& Rescorla, R. A. (1985). Postconditioning devaluation of a reinforcer affects instrumental responding. Journal of Experimental Psychology: Animal Behavior Processes, 11, 120-132.

Colwill, R. M., \& Rescorla, R. A. (1988). Associations between the discriminative stimulus and the reinforcer in instrumental learning. Journal of Experimental Psychology: Animal Behavior Processes, 14, 155-164.

Delamater, A. R. (1996). Effects of several extinction treatments upon the integrity of Pavlovian stimulus-outcome associations. Animal Learning \& Behavior, 24, 437-449.

Dickinson, A., Hall, G., \& Mackintosh, N. J. (1976). Surprise and the attenuation of blocking. Journal of Experimental Psychology: Animal Behavior Processes, 2, 313-322.

GanesAN, R., \& PEARCE, J. M. (1988a). Effect of changing the unconditioned stimulus on appetitive blocking. Journal of Experimental Psychology: Animal Behavior Processes, 14, 280-291.

Ganesan, R., \& Pearce, J. M. (1988b). Interactions between conditioned stimuli for food and water in the rat. Quarterly Journal of Experimental Psychology, 40B, 229-242.

Holland, P. C. (1984). Unblocking in Pavlovian appetitive conditioning. Journal of Experimental Psychology: Animal Behavior Processes, 10, 476-497.

KAMIN, L. J. (1968). Attention-like processes in classical conditioning. In M. R. Jones (Ed.), Miami symposium on the prediction of behavior: Aversive stimulation (pp. 9-32). Chicago: University of Chicago Press.

KAMIN, L. J., \& GAIONI, S. J. (1974). Compound conditioned emotional response conditioning with differentially salient elements in rats. Journal of Comparative \& Physiological Psychology, 87, 591-597.

KONORSKI, J. (1967). Integrative activity of the brain. Chicago: University of Chicago Press.

KREMER, E. F. (1978). The Rescorla-Wagner model: Losses in associative strength in compound conditioned stimuli. Journal of Experimental Psychology: Animal Behavior Processes, 4, 22-36.

LatTAL, K. M., \& NaKaJima, S. (1998). Overexpectation in appetitive Pavlovian and instrumental conditioning. Animal Learning \& Behavior, 26, 351-360.

MackINTOSH, N. J. (1974). The psychology of animal learning. New York: Academic Press.
Pavlov, I. P. (1927). Conditioned reflexes. (G. V. Anrep, Trans.). London: Oxford University Press.

RESCORLA, R. A. (1970). Reduction in the effectiveness of reinforcement after prior excitatory conditioning. Learning \& Motivation, 1 , 327-381.

RESCORLA, R. A. (1995). Full preservation of a response-outcome association through training with a second outcome. Quarterly Journal of Experimental Psychology, 48B, 252-261.

Rescorla, R. A. (1996a). Preservation of Pavlovian associations through extinction. Quarterly Journal of Experimental Psychology, 49B, 245-258.

RESCORLA, R. A. (1996b). Response-outcome associations remain functional through interference treatments. Animal Learning \& Behavior, 24, 450-458.

RESCORLA, R. A. (1996c). Spontaneous recovery after training with multiple outcomes. Animal Learning \& Behavior, 24, 11-18.

ResCoRLA, R. A. (1997a). Spontaneous recovery after Pavlovian conditioning with multiple outcomes. Animal Learning \& Behavior, 25, 99-107.

RESCORLA, R. A. (1997b). Spontaneous recovery of instrumental discriminative responding. Animal Learning \& Behavior, 25, 485-497.

ResCORLA, R. A. (1997c). Summation: Assessment of a configural theory. Animal Learning \& Behavior, 25, 200-209.

ResCORLA, R. A. (1998). Instrumental learning: Nature and persistence. In M. Sabourin, F. I. M. Craik, \& M. Roberts (Eds.), Proceedings of the XXVI International Congress of Psychology: Vol. 2. Advances in psychological science: Biological and cognitive aspects (pp. 239258). London: Psychology Press.

Rescorla, R. A., \& WAGNER, A. R. (1972). A theory of Pavlovian conditioning: Variations in the effectiveness of reinforcement and nonreinforcement. In A. H. Black \& W. F. Prokasy (Eds.), Classical conditioning $I /$ (pp. 64-99). New York: Appleton-Century-Crofts.

WAGNER, A. R. (1971). Elementary associations. In H. H. Kendler \& J. T. Spence (Eds.), Essays in neobehaviorism: A memorial volume to Kenneth W. Spence (pp. 187-213). New York: Appleton-CenturyCrofts.

WATT, A., \& HoNEY, R. C. (1997). Combining CSs associated with the same or different USs. Quarterly Journal of Experimental Psychology, 50B, 350-367.

Williams, B. A. (1994). Blocking despite changes in reinforcer identity. Animal Learning \& Behavior, 22, 442-457.

(Manuscript received February 17, 1998; revision accepted for publication July 18,1998 .) 